University of Nebraska - Lincoln

DigitalCommons@University of Nebraska - Lincoln

$8-2000$

\title{
Theory of Magnetostatic Coupling in Thin-Film Rectangular Magnetic Elements
}

\author{
Evgeny Y. Tsymbal \\ University of Nebraska at Lincoln, tsymbal@unl.edu
}

Follow this and additional works at: https://digitalcommons.unl.edu/physicstsymbal

Part of the Condensed Matter Physics Commons

Tsymbal, Evgeny Y., "Theory of Magnetostatic Coupling in Thin-Film Rectangular Magnetic Elements" (2000). Evgeny Tsymbal Publications. 16.

https://digitalcommons.unl.edu/physicstsymbal/16

This Article is brought to you for free and open access by the Research Papers in Physics and Astronomy at DigitalCommons@University of Nebraska - Lincoln. It has been accepted for inclusion in Evgeny Tsymbal Publications by an authorized administrator of DigitalCommons@University of Nebraska - Lincoln. 


\title{
Theory of magnetostatic coupling in thin-film rectangular magnetic elements
}

\author{
E. Y. Tsymbal ${ }^{\text {a) }}$ \\ Department of Materials, University of Oxford, Parks Road, Oxford OX1 3PH, United Kingdom
}

(Received 18 May 2000; accepted for publication 29 August 2000)

\begin{abstract}
A theory of magnetostatic coupling in thin-film uniformly magnetized arrays of rectangular magnetic elements is presented. Analytic expressions for the magnetostatic energy and the dipolar fields are derived. The influence of the aspect ratio and the spacing between the elements on the magnetostatic coupling is investigated. It is found that increasing the aspect ratio reduces the critical distance between the elements, above which the magnetostatic inter-element coupling can be neglected. (C) 2000 American Institute of Physics. [S0003-6951(00)02343-3]
\end{abstract}

The patterned arrays of submicron magnetic elements have unique magnetic properties, which are distinct from those of the corresponding bulk materials (for the recent review, see Ref. 1 and references therein). These nanostructures are currently attracting increasing interest due to their possible applications in magnetic random access memories ${ }^{2}$ and as advanced replacements for hard disk media. ${ }^{3}$ The memory (storage) density which is one of the most important characteristics of these devices (media) is dependent upon the size and the distance between the bits. The latter quantity is limited by the magnetostatic coupling between the elements. In bulk magnetic materials the magnetostatic coupling is normally small as compared to the exchange interaction. In the array of magnetic elements the exchange interaction between the different elements is absent and therefore the magnetic properties of the array are governed by the magnetostatic coupling. As has been shown both experimentally and theoretically, in patterned magnetic nanostructures the magnetostatic inter-element coupling can play an essential role and strongly affect their magnetic properties. $^{4-7}$

An accurate theoretical treatment of the magnetostatic interactions is based on solving the Landau-Lifshitz-Gilbert torque equation which can be performed using micromagnetic modeling. ${ }^{8}$ Since the magnetic dipolar fields are long ranged and the magnetization is in general inhomogeneous within the element, for an array of magnetic elements this has to involve massive numerical computations. An alternative approach is to assume that the magnetic elements are uniformly magnetized. This approximation allows deriving analytic expressions for the demagnetizing fields and making quantitative conclusions about the influence of the magnetostatic coupling on magnetic properties of nanostructures. Such an approach has been used to analyze minimum-energy magnetic configurations ${ }^{9}$ and to characterize the influence of the magnetostatic coupling on the saturation field. ${ }^{7}$ Reducing the size of the elements down to $0.1 \mu \mathrm{m}$ scale and less makes the magnetic elements single domain, ${ }^{1}$ which results in a better justification for this approach. In this letter we derive exact analytic expressions for the magnetostatic field and en-

${ }^{a)}$ Electronic mail: evgueni.tsymbal@ materials.ox.ac.uk ergy in a periodic array of uniformly magnetized rectangular magnetic elements and investigate the influence of the aspect ratio and the spacing between the elements on the magnetostatic inter-element coupling.

We consider rectangular magnetic elements which are placed in a periodic rectangular lattice, as is shown in Fig. 1. The Cartesian coordinates are set in such a way that the $x$ and $y$ axes lie along the rectangle sides and the $z$ axis lies along the surface normal, as is shown in Fig. 1. The origin of the system is placed in the corner of an element, so that the element occupies the volume $0<x<l, 0<y<d$, and $0<z$ $<h$, where $h$ is the thickness of the element. The magnetization $\mathbf{M}=(0, M, 0)$ is assumed to be homogeneous across the array and oriented along the $y$ direction. The scalar potential which is produced by an infinitely thin layer $d z^{\prime}$ of the array, lying within the $x y$ plane at height $z^{\prime}$, can be found using the following expression: ${ }^{10}$

$$
\begin{aligned}
d \Phi\left(x, y, z-z^{\prime}\right)= & d z^{\prime} \int_{-\infty}^{\infty} d k_{x} \int_{-\infty}^{\infty} d k_{y} \frac{i M k_{y}}{\sqrt{k_{x}^{2}+k_{y}^{2}}} \\
& \times e^{-\sqrt{k_{x}^{2}+k_{y}^{2}}\left|z-z^{\prime}\right|} \tilde{\xi}\left(k_{x}, k_{y}\right) e^{-i k_{x} x} e^{-i k_{y} y},
\end{aligned}
$$

where the Fourier component of the two-dimensional array structure $\xi(x, y)$ is

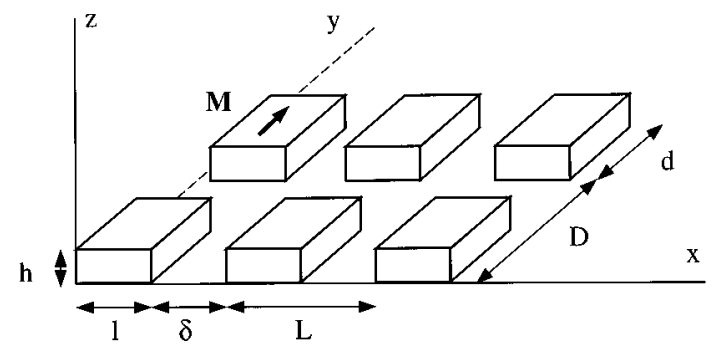

FIG. 1. Geometry of the array of rectangular elements, which was used in calculations. The Cartesian coordinates and the lateral dimensions of the array are shown. 


$$
\begin{aligned}
\tilde{\xi}\left(k_{x}, k_{y}\right) & =\frac{1}{2 \pi} \int_{-\infty}^{\infty} e^{i k_{x} x} d x \int_{-\infty}^{\infty} e^{i k_{y} y} d y \xi(x, y) \\
& =\frac{1}{2 \pi} \sum_{n=-\infty}^{\infty} \int_{n L}^{n L+l} e^{i k_{x} x} d x \sum_{m=-\infty}^{\infty} \int_{m D}^{m D+d} e^{i k_{y} y} d y .
\end{aligned}
$$

By taking into account that

$$
\sum_{n=-\infty}^{\infty} e^{i n k_{x} L}=\frac{2 \pi}{L} \sum_{n=-\infty}^{\infty} \delta\left(k_{x}-\frac{2 \pi}{L} n\right),
$$

Eq. (2) can be rewritten as follows:

$$
\begin{aligned}
\tilde{\xi}\left(k_{x}, k_{y}\right)= & \frac{2 \pi}{L D} \sum_{n=-\infty}^{\infty} \delta\left(k_{x}-\frac{2 \pi}{L} n\right) \frac{e^{i k_{x} l}-1}{i k_{x}} \\
& \times \sum_{m=-\infty}^{\infty} \delta\left(k_{y}-\frac{2 \pi}{D} m\right) \frac{e^{i k_{y} d}-1}{i k_{y}} .
\end{aligned}
$$

Substituting Eq. (4) in Eq. (1) and integrating over $k_{x}$ and $k_{y}$ we obtain

$$
\begin{aligned}
d \Phi\left(x, y, z-z^{\prime}\right)= & \frac{2 \pi M d z^{\prime}}{L D} \sum_{n, m=-\infty}^{\infty} \frac{i k_{2}}{k} \frac{e^{i k_{1} l}-1}{i k_{1}} \\
& \times \frac{e^{i k_{2} d}-1}{i k_{2}} e^{-i k_{1} x} e^{-i k_{2} y} e^{-k\left|z-z^{\prime}\right|},
\end{aligned}
$$

where $k_{1}=2 \pi / L n, k_{2}=2 \pi / D m$ and $k=\sqrt{k_{1}^{2}+k_{2}^{2}}$. In order to calculate the potential of the entire structure, this expression should be integrated over $z^{\prime}$ from 0 to $h$. For $0 \leqslant z \leqslant h$ the result is

$$
\begin{aligned}
\Phi(x, y, z)= & \frac{2 \pi M}{L D} \sum_{n, m=-\infty}^{\infty} \frac{i k_{2}}{k} \frac{e^{i k_{1} l}-1}{i k_{1}} \frac{e^{i k_{2} d}-1}{i k_{2}} \\
& \times e^{-i k_{1} x} e^{-i k_{2} y} \frac{2-e^{-k z}-e^{k(z-h)}}{k} .
\end{aligned}
$$

The magneto-dipolar field can be found by differentiating this expression in accordance with $\mathbf{H}=-\nabla \Phi$. In particular, the $y$ component of the magnetic field, which lies along the magnetization, is

$$
\begin{aligned}
H_{y}(x, y, z)= & -\frac{\partial \Phi}{\partial y} \\
= & \frac{4 \pi M}{L D}\left\{\sum_{m=1}^{\infty} \frac{l}{k_{2}}\left[\sin k_{2}(y-d)-\sin k_{2} y\right]\right. \\
& \times\left[2-e^{-k_{2} z}-e^{k_{2}(z-h)}\right] \\
& -\sum_{n, m=1}^{\infty} \frac{2 k_{2}}{k^{2} k_{1}}\left[\sin k_{1}(x-l)-\sin k_{1} x\right] \\
& \times\left[\sin k_{2}(y-d)-\sin k_{2} y\right] \\
& \left.\times\left[2-e^{-k z}-e^{k(z-h)}\right]\right\} .
\end{aligned}
$$

The magnetostatic energy per unit volume of an element is determined by

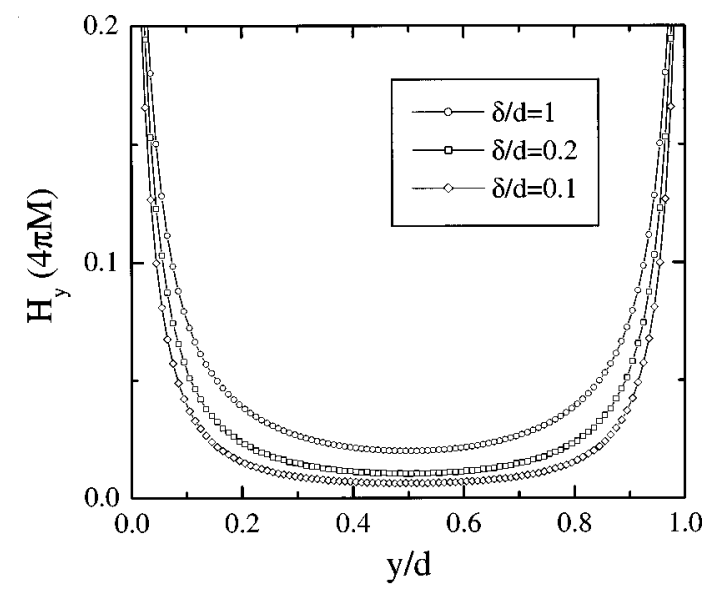

FIG. 2. The profile of the magneto-dipolar field across the element at $x$ $=l / 2$ for the array of square elements, calculated for various distances between the elements $\delta$. The dipolar field is averaged over the thickness of the element $h$. Note that the field is oriented opposite to the magnetization direction.

$$
E=-\frac{1}{2 l} \int_{0}^{l} d x \frac{1}{d} \int_{0}^{d} d y \frac{1}{h} \int_{0}^{h} M H_{y}(x, y, z) d z .
$$

Evaluating this integral we arrive at

$$
\begin{aligned}
E= & \frac{16 \pi M^{2}}{L D l d}\left\{\sum_{m=1}^{\infty} \frac{l^{2}}{k_{2}^{2}} \sin ^{2}\left(\frac{k_{2} d}{2}\right)\left[1-\frac{1-e^{-k_{2} h}}{k_{2} h}\right]\right. \\
& \left.+8 \sum_{n, m=1}^{\infty} \frac{1}{k^{2} k_{1}^{2}} \sin ^{2}\left(\frac{k_{1} l}{2}\right) \sin ^{2}\left(\frac{k_{2} d}{2}\right)\left[1-\frac{1-e^{-k h}}{k h}\right]\right\} .
\end{aligned}
$$

We note that the magnetostatic energy determines an effective demagnetizing field $H_{\text {eff }}$, which can be found from $E=-H_{\text {eff }} M$. This effective field is correlated with the saturation magnetization of the array of single-domain magnetic elements and can therefore be used for the analysis of the hysteresis curves in magnetic nanostructures.

Figure 2 shows the profile of the calculated magnetodipolar field along the magnetization direction at $x=l / 2$ for the array of square elements (i.e., $l=d$ ). The dipolar field was averaged over the thickness of the element $h$, which was set equal to $h=0.05 d$, and was calculated for various distances between the elements $\delta$, which were assumed to be the same in both lateral directions (i.e., $D=d+\delta$ and $L=l$ $+\delta$ ). As is obvious from Fig. 2, the magnetostatic field is very inhomogeneous. It is strongly enhanced in the vicinity of the edges of the elements, reflecting the presence of magnetic poles. This large value of the demagnetizing field facilitates creating the edge domains in the multi-domain elements (e.g., Ref. 7). As is seen from Fig. 2, with increasing the separation between the elements the dipolar field increases. This reflects the fact that the magnetic poles of the opposite sign, which belong to the neighboring elements, become more separated, reducing the diminishing effect on the resulting dipolar field.

Figure 3 shows the calculated magnetostatic energy for the array of the elements with different aspect ratios $d / l$ as a function of the distance between the elements $\delta$. As is evident from the figure, the magnetostatic energy increases with increasing the separation between the elements. The limit of 


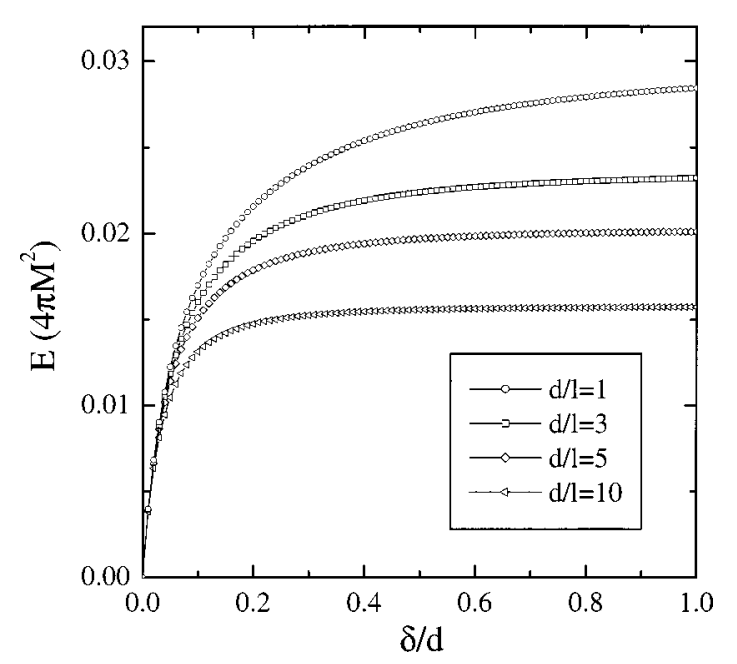

FIG. 3. The calculated magnetostatic energy for the array of the elements with different aspect ratios $d / l$ as a function of the distance between the elements $\delta$. Note that the magnetization is aligned along the long side of the element.

$\delta=0$ corresponds to a nonstructured thin film and in this case the magnetostatic energy equals zero. In the opposite limit of large separations the elements can be considered as noninteracting and the magnetostatic energy is determined by the shape of the individual element. In this limit our results are in good agreement with the predictions of Ref. 11, in which the magneto-dipolar fields of rectangular prisms were calculated. The distance between the elements, at which the magnetostatic energy saturates, corresponds to the vanishing inter-element coupling. With decreasing the separation $\delta$ the magnetostatic energy decreases, reflecting the presence of the magnetostatic coupling between the elements. This decrease in the energy originates from the partial compensation of the demagnetizing fields from the neighboring elements.

As can be seen from Fig. 3, for various aspect ratios $d / l$ of the elements the magnetostatic energy saturates at different separations. For the square-shape elements (the aspect ratio $d / l=1)$ at large separations $\delta$ the magnetostatic coupling is greater than that for the elements with the higher aspect ratio (see Fig. 3). In this case even at $\delta / d=1$ the magnetostatic energy $E=0.28\left(4 \pi M^{2}\right)$ is still less than the saturation energy $E_{\mathrm{sat}}=0.30\left(4 \pi M^{2}\right)$, reflecting the presence of the inter-element coupling. With increasing the aspect ratio the effect of the inter-element coupling becomes less pronounced at large $\delta$. For example, as can be seen from Fig. 3, for $\delta / d=0.3$ the magnetostatic energy is saturated for the elements with the aspect ratio $d / l=10$, whereas it is less than $E_{\text {sat }}$ by $20 \%$ for the elements with the aspect ratio $d / l=1$. Note that for $d / l<1$ the magnetization is aligned along the short side of the element and the magnetostatic interaction becomes stronger.

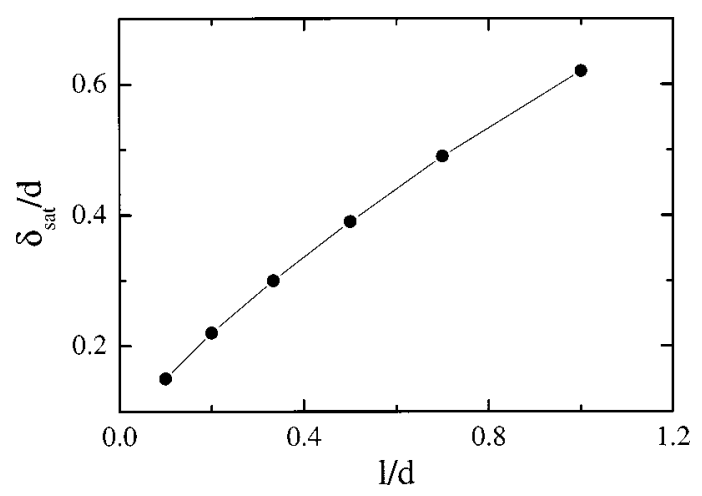

FIG. 4. The separation $\delta_{\text {sat }}$ between the elements, at which the magnetostatic energy saturates, vs the inverse aspect ratio of the rectangular elements $l / d$. The term $\delta_{\text {sat }}$ can serve as a measure of the critical distance, above which the magnetostatic coupling can be neglected.

The separation $\delta_{\text {sat }}$ at which the magnetostatic energy saturates can serve as a measure of the critical distance, above which the magnetostatic coupling can be neglected. The term $\delta_{\text {sat }}$ can be defined as a distance at which $E$ $=0.9 E_{\text {sat }}$ and can then be determined from the energy curves displayed in Fig. 3. The result is shown in Fig. 4. As is evident from the figure, $\delta_{\text {sat }}$ increases almost in a linear fashion as a function of the inverse aspect ratio $l / d$. This implies that for the greater aspect ratios the smaller separations between the rectangular elements are possible, at which the magnetostatic coupling can be neglected. Increasing the aspect ratio of the elements may therefore help to reduce the effect of the magnetostatic coupling in high-density patterned nanostructures.

This research is supported by Hewlett-Packard Laboratories in Palo Alto, CA, through a collaborative research program. The author is thankful to Sergey Demokritov and David Pettifor for the useful discussions.

${ }^{1}$ R. P. Cowburn, J. Phys. D 33, R1 (2000).

${ }^{2}$ S. S. P. Parkin, K. P. Roche, M. G. Samant, P. M. Rice, R. B. Beyers, R. E. Scheuerlein, E. J. O'Sullivan, S. L. Brown, J. Bucchigano, D. W. Abraham, Y. Lu, M. Rooks, P. L. Trouilloud, R. A. Wanner, and W. J. Gallagher, J. Appl. Phys. 85, 5828 (1999).

${ }^{3}$ R. L. White, R. M. H. New, and R. F. W. Pease, IEEE Trans. Magn. 33, 990 (1997).

${ }^{4}$ K. J. Kirk, J. N. Chapman, and C. D. W. Wilkinson, Appl. Phys. Lett. 71, 539 (1997).

${ }^{5}$ T. Aign, P. Meyer, S. Lemerle, J. P. Jamet, J. Ferré, V. Mathet, C. Chappert, J. Gierak, C. Vieu, F. Rousseaux, H. Launois, and H. Bernas, Phys. Rev. Lett. 81, 5656 (1998).

${ }^{6}$ D. Kechrakos and K. N. Trohidou, Phys. Rev. B 58, 12169 (1998).

${ }^{7}$ J. Jorzick, C. Krämer, S. O. Demokritov, B. Hillebrands, E. Sondergard, M. Bailleul, C. Fermon, U. Memmert, A. N. Müller, U. Hartmann, and E. Y. Tsymbal (unpublished).

${ }^{8}$ H. N. Bertram and J.-G. Zhu, in Solid State Physics, edited by H. Ehrenreich and D. Turnbull (Academic, San Diego, 1992), Vol. 46, p. 271.

${ }^{9}$ K. Y. Guslienko, Appl. Phys. Lett. 75, 394 (1999).

${ }^{10}$ E. Y. Tsymbal, J. Magn. Magn. Mater. 130, L6 (1994).

${ }^{11}$ R. I. Joseph and E. Schloemann, J. Appl. Phys. 36, 1679 (1965). 